\title{
Methodology for studying impulse disturbance from rail joints to the railway vehicle and assessment of the efficiency of the new spring suspension
}

\author{
Viktor Nekhaev ${ }^{1}$, Viktor Nikolaev ${ }^{1, *}$, and Evgenii Cheltygmashev ${ }^{1}$ \\ ${ }^{1}$ Omsk State Transport University, 644046, Marx av., 35, Omsk, Russia
}

\begin{abstract}
The methodology for studying the impulse disturbance of the railway track joints on the indicators of the dynamic qualities of the railway vehicle has been developed. The dependence of the impulse repetition factor on the energy dissipation level in the system and the speed of the vehicle is obtained. A comparative assessment of the dynamic qualities of a freight car with a typical scheme of spring suspension and a car with suspension based on the principle of compensation of external disturbances is performed. It has been established that the spring suspension of a freight car based on the principle of compensation of external disturbances delivers to it significantly better indicators of dynamic qualities in comparison with car equipped with a new three-piece truck with a typical scheme of springs. Vertical acceleration of the car's body with a new scheme of vibration protection and dynamic forces in spring suspension is several times less than for a car with a typical three-piece truck structure.
\end{abstract}

\section{Introduction}

The strategy for the development of railway transport involves the expansion of the range of high-speed passenger trains, the creation and organization of high-speed container traffic, as well as the heavy traffic of trains with increased axial and linear loads.

Even when moving along a continuous welded rail track, as shown in [1], due to increased wear and tear in the area of welded joints with the formation of saddles and cracking of the metal particles when the "Sapsan" train moves at a speed of $200 \mathrm{~km} / \mathrm{h}$ every $25 \mathrm{~m}$, i.e. at each welded joint, because of such unevenness, there are bursts on the curves of recording accelerations of the axle boxes (Fig. 1) that reach $80-90 \mathrm{~m} / \mathrm{s}^{2}$.

When the trains move along the continuous welded rail track, the joints of the rails, especially in winter, exert an even higher shock impact on the truck units [2], causing fatigue cracks and fractures, which threaten the safety of train traffic. Also, a large number of disorders of the upper structure of the track and the defects of the rails are accumulated.

* Corresponding author: NikolaevVA@omgups.ru 


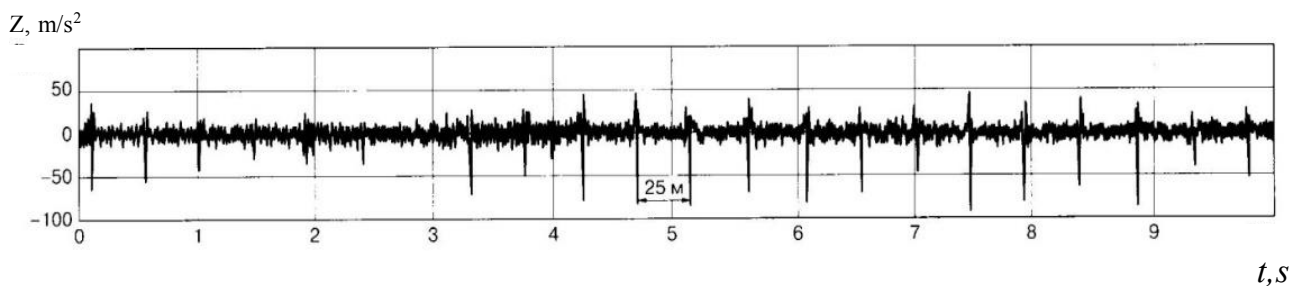

Fig. 1. Vertical acceleration of the axle box of the "Sapsan" train moving along a continuous welded rail track, $\mathrm{V}=200 \mathrm{~km} / \mathrm{h}$.

The annual number of rails withdrawal on the railway network exceeds 102,000 [1]. It is shown in work [3] that in the case when the wheel set hits the junction with the depression of ballast of $10 \mathrm{~mm}$ at a speed of $51 \mathrm{~km} / \mathrm{h}$, the impact force can reach $468 \mathrm{kN}$. The consequence of such high impact loads due to increased stiffness of the track during the winter operation period, large gaps and cracking of metal particles at the ends of rails, and imperfections in casting technology on Russian railways is the large number of cracks in the truck units, leading to their breaks. In 2013 there were 37 breaks in the side frames of freight car trucks with consequent significant negative consequences.

Assessment of the impulse action of an individual wheel with a slider on the track is described in $[4,5]$. The methodology for assessing the impact of cars with sliders in the structure of a moving train on the track was developed by Kogan A.Ya. [6]. At the same time, the task of assessing the effect of the periodic impact of rail joints on the moving railway vehicle has not been solved yet. If we take into account that the share of the continuous welded rail track on the operating domain of the Russian railways remains significant, then the problem arises to develop a methodology for studying impulse disturbance from rail joints to rolling stock, which is also necessary for assessing the efficiency of the newly designed spring suspension of the freight car.

\section{Research methodology}

If we neglect the longitudinal non-equal elasticity of the railway track (this is common for dynamics of rolling stock assumption that allows simplifying the integration of the systems of differential equations of the vehicle), then it is quite possible to use the discrete inertial model of the railway track, since it is quite simple but provides sufficient accuracy. Owing to the longitudinal symmetry of the rolling stock, it is possible to reliably prove that by introducing new "generalized" coordinates, it is possible to reduce the flat design model of the railway vehicle to a conditional single-axle vehicle, the design scheme of which is represented in the form of a system of solids, springs and dampers of dry or viscous friction (Fig.2), can be considered quite justified. The proof of this is also given in [7].

Here the following designations are used: Mk, MT, Mkp, Mp - masses of body, truck, wheel set, and the "reduced" mass of the railway track, respectively, $\mathrm{tf} \cdot \mathrm{s} 2 / \mathrm{m} ; \mathrm{Ck}, \mathrm{Cb}, \mathrm{Cp}$ the stiffness of the central, axle suspension, and the stiffness of the track, which, based on the conditions of our investigation, will be considered constant, tf $/ \mathrm{m} ; \mathrm{bk}, \mathrm{bb}, \mathrm{bp}$ coefficients of equivalent viscous friction of body, axle suspension, and track, $\mathrm{tf} \cdot \mathrm{s} / \mathrm{m}$. All the values mentioned above are calculated either for one wheel set or for one wheel (this is not important!). 


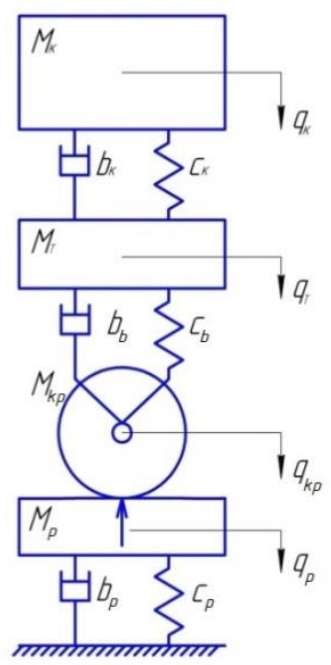

Fig. 2. The design model of the generalized railway vehicle.

Thus, it is obvious that the dynamic behavior of our conditional "generalized" singleaxle railway vehicle is described by a system of ordinary differential equations with constant coefficients written in vector-matrix form:

$$
A \ddot{\vec{q}}+B \dot{\vec{q}}+C \vec{q}=0
$$

where $A=\left(\begin{array}{ccc}M_{k} & 0 & 0 \\ 0 & M_{T} & 0 \\ 0 & 0 & M_{k p}+M_{p}\end{array}\right)$ - matrix of inertial coefficients;

$B=\left(\begin{array}{ccc}b_{k} & -b_{k} & 0 \\ -b_{k} & b_{k}+b_{b} & -b_{b} \\ 0 & -b_{b} & b_{b}+b_{p}\end{array}\right)$ - matrix of dissipative coefficients;

$C=\left(\begin{array}{ccc}C_{k} & -C_{k} & 0 \\ -C_{k} & C_{k}+C_{b} & -C_{b} \\ 0 & -C_{b} & C_{b}+C_{p}\end{array}\right)$ matrix of rigid coefficients;

$\vec{q}=\left(q_{k}, q_{T}, q_{k p}\right)^{T}$ - vector of generalized coordinates; $q_{k}$ - bouncing of a body; $q_{T}$ - bouncing of vehicle's truck; $q_{k p}$ - bouncing of a wheel set.

It should be noted that on the right side of the system of differential equations is zero and, consequently, one might think that the authors are going to study already known things - the natural oscillations of the mechanical system under consideration represented as a conditional single-axle vehicle. But this is not the case as will be shown below.

In some technical issues, it is necessary to deal with short-term forces, the action of which is repeated at relatively large intervals. In these cases, the idea of instantaneous impulses is often used and reduces the problem to the study of the action of periodically applied equal instantaneous impulses I (dimension tf.s). From the course of mechanics, it is known that the impulse is determined by the expression 


$$
I=\int_{0}^{t} u d F_{u d}(t) d t=m\left(V_{+}-V_{-}\right),
$$

where Fud(t) - impact force, which in fact has a different form, tf; tud - time of shock impact, $\mathrm{s} ; \mathrm{m}$ - the mass of the mechanical system on which the impulse acts, $\mathrm{tf} \cdot \mathrm{s} / \mathrm{m} ; \mathrm{V}+$ speed of bouncing of cargo after shock impact, $\mathrm{m} / \mathrm{s} ; \mathrm{V}_{-}$- speed that the cargo had before the shock impact, $\mathrm{m} / \mathrm{s}$. If the time of shock impact (according to the researches of Professor M.P. Pakhomov, the mean impact time in the joint is [8], and the speed of the vehicle changes in $\mathrm{km} / \mathrm{h}$ ) is much less than the period of natural oscillations of the conservative mechanical system, then the criterion of Academician A.N. Krylov [9, 10] allows such a shock with a sufficient degree of accuracy for practical calculations to be considered as instantaneous. This assertion gives the authors the opportunity to refuse taking into account the shape of the shock impulse and to use the known methods of integrating the differential equation for instantaneous periodic impulse action. Indeed, it follows from (2) that the speed of the body after the impact is found from the formula:

$$
V_{+}=V_{-}+\frac{I}{m}
$$

Moreover, we accept the assumptions usual for the theory of impact that the displacement of a solid body cannot instantaneously change because it has inertia, and its speed can increase by the amount of impulse divided by the mass. For a rolling stock moving along a jointed rail track, an expression for the impulse was proposed in [11] for summer and winter operation conditions:

$$
I(V)=\frac{a q+b q^{1 / 3} V}{\omega_{0}\left(1+\delta^{2}\right)}
$$

where $V$ - speed of the vehicle, $\mathrm{km} / \mathrm{h}$, and the regression coefficients of expression (4) are given in the table below, $q$ - unsprung mass of the wheel set, tf, $\omega_{0}=$ $\sqrt{\left(s_{b}+s_{p}\right) /\left(m_{k p}+m_{p}\right)}$ - the natural frequency of the bouncing of a wheel set on an elastic track, $\quad \delta=\left(\beta_{b}+\beta_{p}\right) /\left(2 \sqrt{\left(s_{b}+s_{p}\right)\left(m_{k p}+m_{p}\right)}\right)-$ dimensionless coefficient characterizing the viscous friction in the mechanical system, $s_{b}, s_{p}$ - stiffness of the axle suspension of the vehicle and the railway track, $\mathrm{tf} / \mathrm{m}, \beta_{b}, \beta_{p}$ - coefficients of viscous friction in the axle suspension of the vehicle and the railway track, tf $\cdot \mathrm{s} / \mathrm{m}, m_{k p}, m_{p}$ - mass of the wheel set and the "reduced mass" of the railway track, $\mathrm{tf} \cdot \mathrm{s}^{2} / \mathrm{m}$;

Table1. Values of regression coefficients for periods of the year.

\begin{tabular}{|c|c|c|}
\hline & summer & winter \\
\hline$a$ & 1,654 & 11,47 \\
\hline$b$ & 0,56 & 0,138 \\
\hline
\end{tabular}

The authors of this paper know six ways of solving the problem of the action of instantaneous impulses on a mechanical system [2,5]. All of them are based either on the construction of the corresponding series or on the application of discrete lattice impulse functions proposed by J.Z. Tsypkin, and the penultimate method is the numerical integration of the original system of differential equations. We want to extend the sixth method $[2,5]$ (periodization method of the solution) to mechanical systems with several degrees of freedom. The essence of this method is that the periodic action of impulses on the wheel set during the passage of joints impose its own frequency $2 \pi / T$ on the vibrational mechanical system, here $T=3,6 L_{r} / V\left(L_{r}\right.$ is the length of the rail). 
As is known, the method works well for single-stage conservative mechanical systems. In [6], it was extended to dissipative systems. Next, we will show how to act in the case when the system is multi-stage.

It is known that if it is necessary to single out one single trajectory in a phase space, then it is necessary to specify the initial conditions:

$$
t=0, q_{k}(0)=q_{k 0}, \dot{q}_{k}(0)=\dot{q}_{k 0}, q_{T}(0)=q_{T 0}, \dot{q}_{T}(0)=\dot{q}_{T 0}, q_{k p}(0)=q_{k p 0}, \dot{q}_{k p}(0)=\dot{q}_{k p 0} .
$$

The difference of the mathematical method used by us lies in the fact that these initial conditions must be found from the condition of periodicity of the solution of the mechanical system under study, which is imposed to it by the periodicity of the shock impact. Let's reduce the system (1) to normal coordinates, which give diagonal matrices, in other words, the system breaks up into unconnected separate differential equations, which, as the further mathematics proves, is possible only in the case of two matrices. Nowadays, it's easy to do using any mathematical software package, for example, Mathcad. The eigenvalues of the matrix $A^{-1} C$ are the squares of the partial oscillation frequencies of the mechanical system. Introducing the transformation matrix $\vec{q}=U \vec{x}$, where $\vec{x}$ - the vector of normal coordinates, in our case equal to:

$$
\vec{x}=\left(x_{1}, x_{2}, x_{3}\right)^{T} .
$$

Here the symbol $T$ denotes the transpose of a vector and a matrix. Further, by calculating the matrix $\tilde{C}=U^{-1} A^{-1} C U=\operatorname{diag}\left(k_{01}^{2}, k_{02}^{2}, \ldots, k_{0 n}^{2}\right)$, we see that this is always a diagonal matrix (actually, this is the advantage of introducing normal coordinates). Everything would be fine if the mechanical system were of the conservative type, but we are dealing with a dissipative mechanical system; therefore, we have a matrix $B_{*}=U^{-1} A^{-1} B U$ in which the diagonal elements are much larger than the off-diagonal elements, and then the latter can generally be neglected. It is well known from further mathematics that if there are more than two matrices, then they cannot be reduced to normal coordinates.

In other words, we assume that the matrix of dissipative coefficients is also diagonal and equal to $\tilde{B} \approx \operatorname{diag}\left(2 n_{1}, 2 n_{2}, \ldots, 2 n_{n}\right)$ (this is the only assumption accepted by the authors and, of course, needing further verification), then the original system of differential equations falls into separate subsystems and takes the form:

$$
E \ddot{\vec{x}}+\tilde{B} \dot{\vec{x}}+C \vec{x}=0
$$

here $E=\operatorname{diag}(1,1, \ldots, 1)$ - identity matrix. In the case under consideration, we have:

$$
\left\{\begin{array}{l}
\ddot{x}_{1}+2 n_{1} \dot{x}_{1}+k_{01}^{2} x_{1}=0 \\
\ddot{x}_{2}+2 n_{2} \dot{x}_{2}+k_{02}^{2} x_{2}=0 \\
\ddot{x}_{3}+2 n_{3} \dot{x}_{3}+k_{03}^{2} x_{3}=0
\end{array}\right.
$$

Each of the equations of this system is easily integrated and, consequently, the solution can be written in the form:

$$
x_{j}(t)=e^{-n_{j} t}\left(A_{j} \cos k_{o j} t+B_{j} \sin k_{o j} t\right),
$$

where coefficients $A_{j}$ and $B_{j}$ depend on the initial conditions. Let's write the solution of the original system of differential equations (1) in terms of normal coordinates: 


$$
\left\{\begin{array}{l}
q_{1}(t)=\sum_{j=1}^{3} u_{1, j} e^{-n_{j} t}\left(A_{j} \cos k_{0 j} t+B_{j} \sin k_{0 j} t\right) \\
q_{2}(t)=\sum_{j=1}^{3} u_{2, j} e^{-n_{j} t}\left(A_{j} \cos k_{0 j} t+B_{j} \sin k_{0 j} t\right) \\
q_{3}(t)=\sum_{j=1}^{3} u_{3, j} e^{-n_{j} t}\left(A_{j} \cos k_{0 j} t+B_{j} \sin k_{0 j} t\right)
\end{array}\right.
$$

and represent this result in vector-matrix form:

$$
\vec{q}(t)=\Omega_{c}(t) \vec{A}+\Omega_{s}(t) \vec{B},
$$

where $\vec{A}=\left(A_{1}, A_{2}, A_{3}\right)^{T} ; \vec{B}=\left(B_{1}, B_{2}, B_{3}\right)^{T}$ - vectors of integration constants, representing the amplitude of the oscillations of normal coordinates;

$$
\begin{aligned}
& \Omega_{c}(t)=\left(\begin{array}{lll}
U_{1,1} e^{-n_{1} t} \cos k_{01} t & U_{1,2} e^{-n_{2} t} \cos k_{02} t & U_{1,3} e^{-n_{3} t} \cos k_{03} t \\
U_{2,1} e^{-n_{1} t} \cos k_{01} t & U_{2,2} e^{-n_{2} t} \cos k_{02} t & U_{2,3} e^{-n_{3} t} \cos k_{03} t \\
U_{3,1} e^{-n_{1} t} \cos k_{01} t & U_{3,2} e^{-n_{2} t} \cos k_{02} t & U_{3,3} e^{-n_{3} t} \cos k_{03} t
\end{array}\right) ; \\
& \Omega_{s}(t)=\left(\begin{array}{lll}
U_{1,1} e^{-n_{1} t} \sin k_{01} t & U_{1,2} e^{-n_{2} t} \sin k_{02} t & U_{1,3} e^{-n_{3} t} \sin k_{03} t \\
U_{2,1} e^{-n_{1} t} \sin k_{01} t & U_{2,2} e^{-n_{2} t} \sin k_{02} t & U_{2,3} e^{-n_{3} t} \sin k_{03} t \\
U_{3,1} e^{-n_{1} t} \sin k_{01} t & U_{3,2} e^{-n_{2} t} \sin k_{02} t & U_{3,3} e^{-n_{3} t} \sin k_{03} t
\end{array}\right) .
\end{aligned}
$$

Since we need to determine the six integration constants, it is necessary to differentiate (9) in time, then we obtain:

$$
\left\{\begin{array}{l}
\dot{q}_{1}(t)=\sum_{j=1}^{3} u_{1, j} e^{-n_{j} t}\left[-n_{j}\left(A_{j} \cos k_{0 j} t+B_{j} \sin k_{0 j} t\right)+\right. \\
\left.+k_{0 j}\left(-A_{j} \sin k_{0 j} t+B_{j} \cos k_{0 j} t\right)\right] \\
\dot{q}_{2}(t)=\sum_{j=1}^{3} u_{2, j} e^{-n_{j} t}\left[-n_{j}\left(A_{j} \cos k_{0 j} t+B_{j} \sin k_{0 j} t\right)+\right. \\
\left.+k_{0 j}\left(-A_{j} \sin k_{0 j} t+B_{j} \cos k_{0 j} t\right)\right] \\
\dot{q}_{3}(t)=\sum_{j=1}^{3} u_{3, j} e^{-n_{j} t}\left[-n_{j}\left(A_{j} \cos k_{0 j} t+B_{j} \sin k_{0 j} t\right)+\right. \\
\left.+k_{0 j}\left(-A_{j} \sin k_{0 j} t+B_{j} \cos k_{0 j} t\right)\right]
\end{array}\right.
$$

Analogously to the above calculations, we can write the following vector-matrix form of the solution:

$$
\begin{aligned}
& \dot{\vec{q}}(t)=U \dot{\vec{x}}(t)=-\Omega_{n c}(t) \vec{A}-\Omega_{n s}(t) \vec{B}-\Omega_{k s}(t) \vec{A}+\Omega_{k c}(t) \vec{B}= \\
& =-\left[\Omega_{n c}(t)+\Omega_{k s}(t)\right] \vec{A}-\left[\Omega_{n s}(t)-\Omega_{k c}(t)\right] \vec{B},
\end{aligned}
$$

here 


$$
\begin{aligned}
& \Omega_{n c}(t)=\left(\begin{array}{lll}
U_{1,1} n_{1} e^{-n_{1} t} \cos k_{01} t & U_{1,2} n_{2} e^{-n_{2} t} \cos k_{02} t & U_{1,3} n_{3} e^{-n_{3} t} \cos k_{03} t \\
U_{2,1} n_{1} e^{-n_{1} t} \cos k_{01} t & U_{2,2} n_{2} e^{-n_{2} t} \cos k_{02} t & U_{2,3} n_{3} e^{-n_{3} t} \cos k_{03} t \\
U_{3,1} n_{1} e^{-n_{1} t} \cos k_{01} t & U_{3,2} n_{2} e^{-n_{2} t} \cos k_{02} t & U_{3,3} n_{3} e^{-n_{3} t} \cos k_{03} t
\end{array}\right) \\
& \Omega_{n s}(t)=\left(\begin{array}{lll}
U_{1,1} n_{1} e^{-n_{1} t} \sin k_{01} t & U_{1,2} n_{2} e^{-n_{2} t} \sin k_{02} t & U_{1,3} n_{3} e^{-n_{3} t} \sin k_{03} t \\
U_{2,1} n_{1} e^{-n_{1} t} \sin k_{01} t & U_{2,2} n_{2} e^{-n_{2} t} \sin k_{02} t & U_{2,3} n_{3} e^{-n_{3} t} \sin k_{03} t \\
U_{3,1} n_{1} e^{-n_{1} t} \sin k_{01} t & U_{3,2} n_{2} e^{-n_{2} t} \sin k_{02} t & U_{3,3} n_{3} e^{-n_{3} t} \sin k_{03} t
\end{array}\right) \\
& \Omega_{k c}(t)=\left(\begin{array}{lll}
U_{1,1} k_{01} e^{-n_{1} t} \cos k_{01} t & U_{1,2} k_{02} e^{-n_{2} t} \cos k_{02} t & U_{1,3} k_{03} e^{-n_{3} t} \cos k_{03} t \\
U_{2,1} k_{01} e^{-n_{1} t} \cos k_{01} t & U_{2,2} k_{02} e^{-n_{2} t} \cos k_{02} t & U_{2,3} k_{03} e^{-n_{3} t} \cos k_{03} t \\
U_{3,1} k_{01} e^{-n_{1} t} \cos k_{01} t & U_{3,2} k_{02} e^{-n_{2} t} \cos k_{02} t & U_{3,3} k_{03} e^{-n_{3} t} \cos k_{03} t
\end{array}\right) ; \\
& \Omega_{k s}(t)=\left(\begin{array}{lll}
U_{1,1} k_{01} e^{-n_{1} t} \sin k_{01} t & U_{1,2} k_{02} e^{-n_{2} t} \sin k_{02} t & U_{1,3} k_{03} e^{-n_{3} t} \sin k_{03} t \\
U_{2,1} k_{01} e^{-n_{1} t} \sin k_{01} t & U_{2,2} k_{02} e^{-n_{2} t} \sin k_{02} t & U_{2,3} k_{03} e^{-n_{3} t} \sin k_{03} t \\
U_{3,1} k_{01} e^{-n_{1} t} \sin k_{01} t & U_{3,2} k_{02} e^{-n_{2} t} \sin k_{02} t & U_{3,3} k_{03} e^{-n_{3} t} \sin k_{03} t
\end{array}\right) .
\end{aligned}
$$

Substituting the initial conditions of the problem in (10) and (14), we find the constants of integration from the system of equations:

$$
\left\{\begin{array}{l}
\vec{q}(0)=\vec{q}_{0}=\Omega_{c}(0) \vec{A} ; \\
\dot{\vec{q}}(0)=\dot{\vec{q}}_{0}=-\Omega_{n c}(0) \vec{A}+\Omega_{k c}(0) \vec{B},
\end{array}\right.
$$

it follows that:

$$
\left\{\begin{array}{l}
\vec{A}=U^{-1} \cdot \vec{q}_{0} ; \\
\vec{B}=\Omega_{k c}^{-1}(0) \cdot E \cdot \dot{\vec{q}}_{0}+\Omega_{k c}^{-1}(0) \cdot \Omega_{c}^{-1}(0) \cdot \vec{q}_{0} .
\end{array}\right.
$$

As a result of the above actions, we expressed the constants of integrating the system of differential equations (1) in terms of given but not yet known initial conditions that must be determined using the method of periodization of the solution under an impulse action on the mechanical system. Combining expressions (9) and (13), (10) and (14), we obtain:

$$
\begin{aligned}
& \left\{\begin{array}{l}
\vec{q}(t)=U \cdot \vec{x}(t) ; \\
\dot{\vec{q}}(t)=U \cdot \dot{\vec{x}}(t) ;
\end{array}\right. \\
& \left\{\begin{array}{l}
\vec{q}(t)=\Omega_{c}(t) \cdot \vec{A}+\Omega_{s}(t) \cdot \vec{B} ; \\
\dot{\vec{q}}(t)=-\left[\Omega_{n c}(t)+\Omega_{k s}(t)\right] \cdot \vec{A}-\left[\Omega_{n s}(t)-\Omega_{k c}(t)\right] \cdot \vec{B} .
\end{array}\right.
\end{aligned}
$$

We substitute the corresponding expressions from (20) into formula (22), and after simple transformations we have:

$$
\left\{\begin{array}{l}
\vec{q}(t)=\left[\Omega_{c}(t) \cdot \Omega_{c}^{-1}(0)+\Omega_{s}(t) \cdot \Omega_{k c}^{-1}(0) \cdot \Omega_{c}^{-1}(0)\right] \cdot \vec{q}_{0}+ \\
+\Omega_{s}(t) \cdot \Omega_{k c}^{-1}(0) \cdot E \cdot \dot{\vec{q}}_{0} \\
\dot{\vec{q}}(t)=\left\{-\left[\Omega_{n c}(t)+\Omega_{k s}(t)\right] \cdot \Omega_{c}^{-1}(0)-\left[\Omega_{n s}(t)-\Omega_{k c}(t)\right] \cdot\right. \\
\left.\cdot \Omega_{k c}^{-1}(0) \cdot \Omega_{c}^{-1}(0)\right\} \cdot \vec{q}_{0}-\left[\Omega_{n s}(t)-\Omega_{k c}(t)\right] \cdot \Omega_{k c}^{-1}(0) \cdot E \cdot \dot{\vec{q}}_{0} .
\end{array}\right.
$$


According to the first expression (21), as well as the generally accepted assumptions in the theory of impact, the displacements cannot instantly change, since the bodies have inertial properties and the speed of bouncing a wheel set increases by an amount determined by the impulse.

So, at the end of the period of the oscillations of the wheel set just before applying the next impulse, we have that the vector of bouncing the body, the truck, and the wheel set is:

$$
\vec{q}(T)=U \cdot \vec{x}(T)
$$

but, immediately after applying the next impulse, the displacement $\vec{q}(T)$ will retain its value, and, according to the assumed assumptions for an instantaneous impact, it should be equal to the initial value, i.e.:

$$
\vec{q}(T)=\vec{q}_{0},
$$

since this should correspond to the method of periodization of the solution. The speed of the bouncing of the wheel set will instantly change by the value $I(V) / M_{k p}$, which can be expressed as follows:

$$
\dot{\vec{q}}(T)=U \cdot \dot{\vec{x}}(T)+\frac{1}{M_{k p}} \vec{I}(V)=\dot{\vec{q}}_{0},
$$

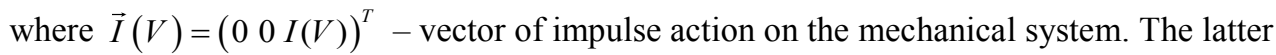
equation also meets the requirement of the method of periodization of the solution.

Thus, equations (25) and (26) form a system of algebraic equations (SAE) with respect to the so far unknown initial conditions of the system of differential equations (1), which we obtain after simple transformations in this form:

$$
H(T) \cdot \vec{z}=\vec{Q}(V),
$$

here $\vec{z}=\left(q_{01}, q_{02}, q_{03}, \dot{q}_{01}, \dot{q}_{02}, \dot{q}_{03}\right)^{T}$ - vector of unknown initial conditions of the system of differential equations $(1) ; \vec{Q}(V)=-\frac{1}{M_{k p}}(0,0,0,0,0, I(V))^{T}-$ vector of right parts of SAE;

$$
\begin{gathered}
H(T)=\left(\begin{array}{ll}
h_{1,1}(T) & h_{1,2}(T) \\
h_{2,1}(T) & h_{2,2}(T)
\end{array}\right)-\text { a square matrix of } 6 \times 6 \\
\left\{\begin{array}{l}
h_{1,1}(T)=\Omega_{c}(T) \cdot \Omega_{c}^{-1}(0)+\Omega_{s}(T) \cdot \Omega_{k c}^{-1}(0) \cdot \Omega_{c}^{-1}(0)-E \\
h_{1,2}(T)=\Omega_{s}(T) \cdot \Omega_{k c}^{-1}(0) \cdot E \\
h_{2,1}(T)=-\left[\Omega_{n c}(T)+\Omega_{k s}(T)\right] \cdot \Omega_{c}^{-1}(0)-\left[\Omega_{n s}(T)-\Omega_{k c}(T)\right] \cdot \Omega_{k c}^{-1}(0) \cdot \Omega_{c}^{-1}(0) ; \\
h_{2,2}(T)=-\left\{\left[\Omega_{n s}(T)-\Omega_{k c}(T)\right] \cdot \Omega_{k c}^{-1}(0) \cdot E+E\right\}
\end{array}\right.
\end{gathered}
$$

an auxiliary matrix of $3 \times 3$.

Thus, we have constructed a methodology for studying instantaneous periodic shock impulses acting on multi-step mechanical systems by the periodization method of the solution. Solving SAE (27) under different conditions, for example, when the stiffness of the railway track, the speed of the vehicle, the period of the year and others are changed, we determine the initial conditions of the system (1). Then, using the whole mathematical baggage of the theory of oscillations of mechanical systems, we can construct the necessary 
graphs of the corresponding task variables, including the bounce curves of the body, the truck, and the wheel set, their impulse repetition rates, bouncing accelerations of the body, the truck, and the wheel set, forces in central and axle stages of spring suspension and in contact of the wheel and rail.

Because of the fact that later we will need comparative estimates of the acceleration of the bouncing of the body, the truck, and the wheel set, we take the time derivative of the second expression (21) and obtain:

$$
\ddot{\vec{q}}(t)=U \cdot \ddot{\vec{x}}(t)
$$

where

$$
\ddot{\vec{x}}(t)=\left(\begin{array}{l}
e^{-n_{1} t}\left[\left(n_{1}^{2}-k_{01}^{2}\right)\left(A_{1} \cos k_{01} t+B_{1} \sin k_{01} t\right)-\right. \\
\left.-2 n_{1} k_{01}\left(-A_{1} \sin k_{01} t+B_{1} \cos k_{01} t\right)\right] \\
e^{-n_{2} t}\left[\left(n_{2}^{2}-k_{02}^{2}\right)\left(A_{2} \cos k_{02} t+B_{2} \sin k_{02} t\right)-\right. \\
\left.-2 n_{2} k_{02}\left(-A_{2} \sin k_{02} t+B_{2} \cos k_{02} t\right)\right] \\
e^{-n_{3} t}\left[\left(n_{3}^{2}-k_{03}^{2}\right)\left(A_{3} \cos k_{03} t+B_{3} \sin k_{03} t\right)-\right. \\
\left.-2 n_{3} k_{03}\left(-A_{3} \sin k_{03} t+B_{3} \cos k_{03} t\right)\right]
\end{array}\right) .
$$

Formula (29) taking into account expression (30) can be reduced to the form represented by relation (14), but this is not necessary, since expressions (29) and (30) are sufficient. And yet we write down these relations:

$$
\ddot{\vec{q}}(t)=\left[L_{1}(t)+L_{3}(t)\right] \cdot \vec{A}+\left[L_{2}(t)-L_{4}(t)\right] \cdot \vec{B},
$$

Where

$$
\begin{gathered}
L_{1}(t)=\left(\begin{array}{lll}
U_{1,1} e^{-n_{1} t}\left(n_{1}^{2}-k_{01}^{2}\right) \cos k_{01} t & U_{1,2} e^{-n_{2} t}\left(n_{2}^{2}-k_{02}^{2}\right) \cos k_{02} t & U_{1,3} e^{-n_{3} t}\left(n_{3}^{2}-k_{03}^{2}\right) \cos k_{03} t \\
U_{2,1} e^{-n_{1} t}\left(n_{1}^{2}-k_{01}^{2}\right) \cos k_{01} t & U_{2,2} e^{-n_{2} t}\left(n_{2}^{2}-k_{02}^{2}\right) \cos k_{02} t & U_{2,3} e^{-n_{3} t}\left(n_{3}^{2}-k_{03}^{2}\right) \cos k_{03} t \\
U_{3,1} e^{-n_{1} t}\left(n_{1}^{2}-k_{01}^{2}\right) \cos k_{01} t & U_{3,2} e^{-n_{2} t}\left(n_{2}^{2}-k_{02}^{2}\right) \cos k_{02} t & U_{3,3} e^{-n_{3} t}\left(n_{3}^{2}-k_{03}^{2}\right) \cos k_{03} t
\end{array}\right) ; \\
L_{2}(t)=\left(\begin{array}{lll}
U_{1,1} e^{-n_{1} t}\left(n_{1}^{2}-k_{01}^{2}\right) \sin k_{01} t & U_{1,2} e^{-n_{2} t}\left(n_{2}^{2}-k_{02}^{2}\right) \sin k_{02} t & U_{1,3} e^{-n_{3} t}\left(n_{3}^{2}-k_{03}^{2}\right) \sin k_{03} t \\
U_{2,1} e^{-n_{1} t}\left(n_{1}^{2}-k_{01}^{2}\right) \sin k_{01} t & U_{2,2} e^{-n_{2} t}\left(n_{2}^{2}-k_{02}^{2}\right) \sin k_{02} t & U_{2,3} e^{-n_{3} t}\left(n_{3}^{2}-k_{03}^{2}\right) \sin k_{03} t \\
U_{3,1} e^{-n_{1} t}\left(n_{1}^{2}-k_{01}^{2}\right) \sin k_{01} t & U_{3,2} e^{-n_{2} t}\left(n_{2}^{2}-k_{02}^{2}\right) \sin k_{02} t & U_{3,3} e^{-n_{3} t}\left(n_{3}^{2}-k_{03}^{2}\right) \sin k_{03} t
\end{array}\right) ; \\
L_{4}(t)=\left(\begin{array}{lll}
2 U_{1,1} e^{-n_{1} t} n_{1} k_{01} \cos k_{01} t & 2 U_{1,2} e^{-n_{2} t} n_{2} k_{02} \cos k_{02} t & 2 U_{1,3} e^{-n_{3} t} n_{3} k_{03} \cos k_{03} t \\
2 U_{2,1} e^{-n_{1} t} n_{1} k_{01} \cos k_{01} t & 2 U_{2,2} e^{-n_{2} t} n_{2} k_{02} \cos k_{02} t & 2 U_{2,3} e^{-n_{3} t} n_{3} k_{03} \cos k_{03} t \\
2 U_{3,1} e^{-n_{1} t} n_{1} k_{01} \cos k_{01} t & 2 U_{3,2} e^{-n_{2} t} n_{2} k_{02} \cos k_{02} t & 2 U_{3,3} e^{-n_{3} t} n_{3} k_{03} \cos k_{03} t
\end{array}\right) .
\end{gathered}
$$

As is well known [2,5], a very important characteristic of shock processes is influence coefficient of the impulse repetition, which was found by G. G. Panovko [2] for a conservative system, i.e. when $b=0, n=0, \delta=0$. In this case, for the influence coefficient of the impulse repetition shown in Fig. 3, we have 


$$
\beta=\frac{1}{2\left|\sin \frac{k_{0} T \mid}{2}\right|} .
$$

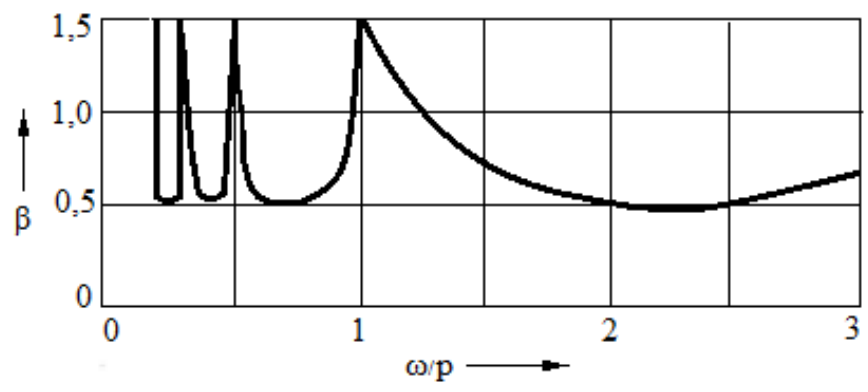

Fig. 3. The influence coefficient of the impulse repetition of a conservative system.

As can be seen from this figure, an unlimited number of shock resonances are possible here. The smallest possible value of $\beta$ is $1 / 2$. The angular frequency of impulse application is the following expression: $\omega=2 \pi / T$.

In work [12], the following formula for the influence coefficient of the impulse repetition was derived for the dissipative system:

$$
\beta=\sqrt{\frac{e^{2 \pi \delta \lambda}}{2(\operatorname{ch} 2 \pi \delta \lambda-\cos 2 \pi \lambda)}},
$$

where $\lambda=T / T_{0}$ - frequency detuning of the system; $T_{0}=2 \pi / k_{0}$ - period of natural oscillations of the system; $\delta=n / k_{0}$ - dimensionless coefficient of viscous friction, the study of which allows establishing that shock resonances can also develop in a dissipative system. This phenomenon is possible when the denominator of the influence coefficient of the impulse repetition is zero, i.e.

$$
\operatorname{ch} 2 \pi \delta \lambda-\cos 2 \pi \lambda=0 .
$$

The graph of the variation of the influence coefficient of the impulse repetition (33) for a dissipative system is shown in Fig. 4. It follows, first, that shock resonances can occur when $\lambda$ is equal to $0,1,2,3$, and so on, and secondly, due to the presence of friction in the system, the maximum values of $\beta$ are limited, while the increase in friction leads to an increase in the minimum of this indicator. However, when we are dealing with multi-stage mechanical systems, it is very difficult (and sometimes it is impossible) to obtain for them a coefficient that stands before the amplitude of the bouncing of the railway vehicle's units. Nevertheless, we will construct an algorithm for calculating the repetition coefficients for rolling stock units.

Let us make these calculations for a conditional single-axel car (locomotive), which can easily be extended to mechanical systems with a large number of degrees of freedom. So, we have:

$$
\left\{\begin{array}{l}
q_{10}=U_{1,1} x_{1}(0)+U_{1,2} x_{2}(0) ; \\
q_{20}=U_{2,1} x_{1}(0)+U_{2,2} x_{2}(0),
\end{array}\right.
$$


here $x_{1}(0)=A_{1} ; x_{2}(0)=A_{2}$. If we assume that there were no vertical oscillations of the wheel set before the shock at the joint, i.e. $q_{10}=q_{20}=0$, then it is easy to establish that the system of algebraic equations (35) is satisfied by a trivial solution, hence $A_{1}=A_{2}=0$.

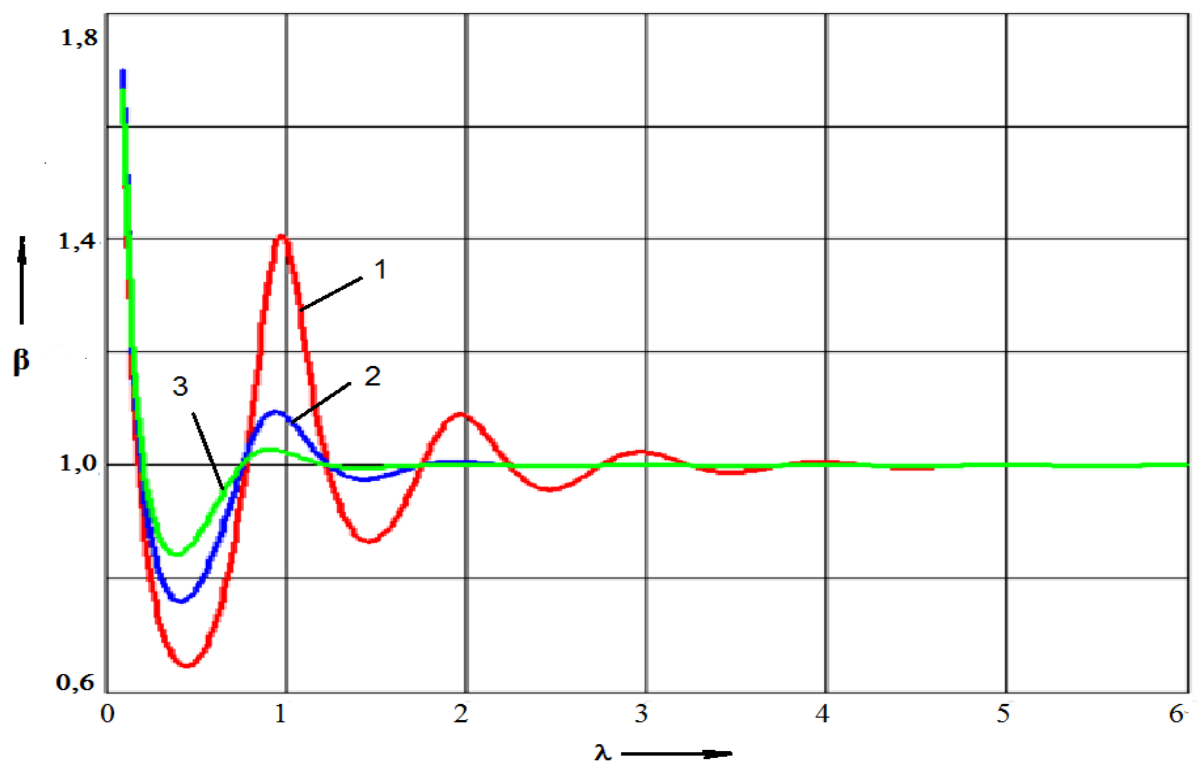

Fig. 4. The influence coefficient of the impulse repetition for the vehicle in question with energy dissipation. Where $1-\delta=0,2 ; 2-\delta=0,4 ; 3-\delta=0,6$.

For the speeds of bouncing, we write:

$$
\left\{\begin{array}{l}
\dot{q}_{1}(t)=U_{1,1} \dot{x}_{1}(t)+U_{1,2} \dot{x}_{2}(t) ; \\
\dot{q}_{2}(t)=U_{2,1} \dot{x}_{1}(t)+U_{2,2} \dot{x}_{2}(t),
\end{array}\right.
$$

or

$$
\left\{\begin{array}{l}
\dot{q}_{10}=U_{1,1} \dot{x}_{1}(0)+U_{1,2} \dot{x}_{2}(0) \\
\dot{q}_{20}=U_{2,1} \dot{x}_{1}(0)+U_{2,2} \dot{x}_{2}(0)
\end{array}\right.
$$

where

$$
\left\{\begin{array}{l}
\dot{x}_{1}(0)=-n_{1} A_{1}+k_{01} B_{1} ; \\
\dot{x}_{2}(0)=-n_{2} A_{2}+k_{02} B_{2} .
\end{array}\right.
$$

Substitution of relations (38) into expressions (37) gives us a system of algebraic equations with respect to the variables $B_{1}$ and $B_{2}$, whose roots are equal to:

$$
\left\{\begin{array}{l}
B_{1}=-\frac{U_{1,2}}{\Delta} \frac{I(V)}{m_{k n} k_{01}} ; \\
B_{2}=\frac{U_{1,1}}{\Delta} \frac{I(V)}{m_{k n} k_{02}} ; \\
\Delta=k_{01} k_{02}\left|\begin{array}{ll}
U_{1,1} & U_{1,2} \\
U_{2,1} & U_{2,2}
\end{array}\right| .
\end{array}\right.
$$


It should be noted that in this case we took into account the equality of the amplitudes $A_{1}$ and $A_{2}$ to zero and the condition of shock impact on the wheel set, from which it follows that $\dot{q}_{10} \equiv 0$ and $\dot{q}_{20}=I(V) / m_{k n}$.

So, we have:

$$
\begin{gathered}
\vec{x}=\left(\begin{array}{c}
B_{1} e^{-n_{1} t} \sin k_{01} t \\
B_{2} e^{-n_{2} t} \sin k_{02} t
\end{array}\right) \\
\dot{\vec{x}}(t)=\left(\begin{array}{c}
B_{1} e^{-n_{1} t}\left(-n_{1} \sin k_{01} t+k_{01} \cos k_{01} t\right) \\
B_{2} e^{-n_{2} t}\left(-n_{2} \sin k_{02} t+k_{02} \cos k_{02} t\right)
\end{array}\right) ; \\
\ddot{\vec{x}}(t)=\left(\begin{array}{c}
B_{1} e^{-n_{1} t}\left[\left(n_{1}^{2}-k_{01}^{2}\right) \sin k_{01} t-2 n_{1} k_{01} \cos k_{01} t\right] \\
B_{2} e^{-n_{2} t}\left[\left(n_{2}^{2}-k_{02}^{2}\right) \sin k_{02} t-2 n_{2} k_{02} \cos k_{02} t\right]
\end{array}\right) ;
\end{gathered}
$$

and

$$
\left\{\begin{array}{l}
\vec{q}(t)=U \cdot \vec{x}(t) \\
\dot{\vec{q}}(t)=U \cdot \dot{\vec{x}}(t) \\
\ddot{\vec{q}}(t)=U \cdot \ddot{\vec{x}}(t) .
\end{array}\right.
$$

Considering first the periodic action of the impulses on the wheel set of the vehicle, and then its single effects on the rolling stock, we find the maximum values in these two arrays, assuming that their ratio gives us the influence coefficient of the impulse repetition, which can be represented in the graph depending on speed of the vehicle.

\section{The results of applying the methodology for assessing the efficiency of new technical solutions}

For a long time, many scientific organizations and educational institutions of higher education in Russia and other countries have been engaged in the development of freight car trucks. Many technical solutions of previous years adopted abroad are repeated in the designs of three-piece trucks. Since the beginning of the two thousand year, about 20 modifications of three-piece trucks have been developed in the countries of Russia and Ukraine. These trucks have different deign of the axle box or adapter, wear-resistant element of the axle unit, side frame, suspension springs, friction wedges, bolster, side slips and are unified only by wheel sets, cassette bearings, and brake gear.

New solutions are mainly associated with the introduction of elastic braces and polyurethane elements between the axle frame and the side frame of the truck (truck 181711), wear-resistant elements in friction pairs and contact slips (for example, truck 18-9855 Barber S-2-R). Here it is necessary to note that all these truck are out of date, have a significant unsprung mass, which has a significant dynamic impact on the track.

As shown in $[14,15]$, a comprehensive solution of the problem of increasing the dynamic qualities of a freight car can be achieved by creating an axle-based sprung stage based on the application of the principle of compensation for external disturbances.

We will use the methodology for studying the impact shock disturbance on a railway rolling stock developed in this paper to study the dynamic behavior of a freight car with a typical suspension scheme: a static deflection of $70 \mathrm{~mm}$ and an axial load of 25 tons/axis (like a Barber S-2-R 18-9855 truck) and car with spring suspension of the axle stage, based 
on the principle of compensation of external disturbances and developed by the staff of the Department of Theoretical Mechanics of OmSTU, with the same axial load.

The impulse action model was chosen according to [3]. Dependences of the vertical acceleration of the body of a laden car with a typical three-piece truck (1) and a truck with a spring suspension developed by the Omsk State Transport University (2) are shown in Fig.5.

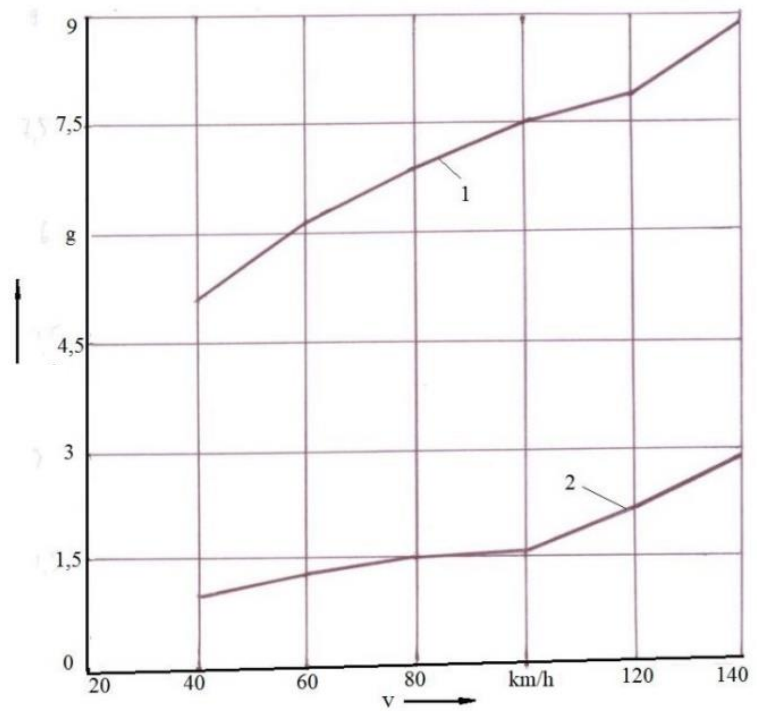

Fig. 5. Vertical acceleration of the body of a laden car with a typical three-piece truck (1) and a truck with suspension developed by OmSTU (2).

Figure 6 reflects the effect of the scheme solutions of the spring system of the vehicle on the level of dynamic forces in the spring suspensions of the compared cars in the laden mode at various speeds of movement along the jointed track in winter.

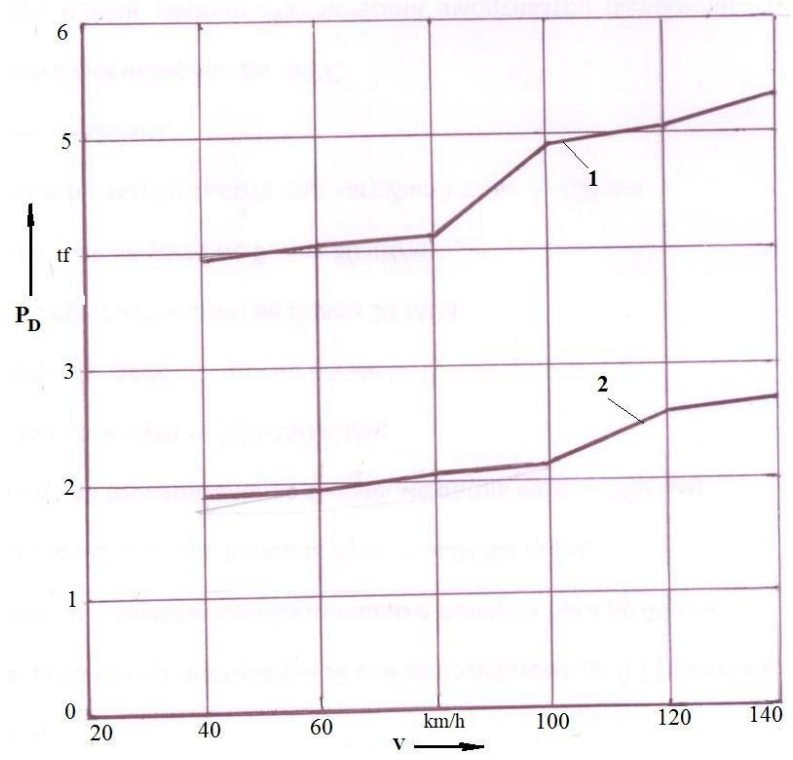

Fig. 6. Dynamic forces in spring packs of standard three-piece truck (1) and in a truck with suspension developed by OmSTU (2). 


\section{Conclusions}

The described methodology for studying the impulse disturbance on a rolling stock gives the chance to assess efficiency of new technical solutions on indicators of dynamic qualities of railway vehicles, including on dynamic loading of units of the truck. The above dependences show a significant improvement in the dynamic properties of a car with suspension based on the principle of compensating external disturbances: the vertical acceleration of the car body with a new spring suspension is several times smaller than that of a car with a typical spring set having a deflection of $70 \mathrm{~mm}$; the dynamic forces in suspension, which determine the loading of the frame of the truck and the impact on the track, are also 2.5-3 times smaller.

So, the system of springs of a freight car's truck, based on the principle of compensating external disturbances, provides it with significantly better dynamic qualities. This will contribute to improving the safety of train traffic, reducing the dynamic loading of the car components and the upper structure of the track, as well as improving the smoothness of the cars' movement, i.e. improving the operational performance and competitiveness of the railway transport.

\section{References}

1. S.V. Khlyst, V.M. Kuzmichenko, V.A. Rezanov, A.I. Borts, E.A. Shur, Vestnik VNIIZhTa, 3, 14 -20 (2013)

2. M.P. Pakhomov, N.P. Buynova, I.I. Galiev, Proceedings of the Omsk Institute of Railway Transport Engineers, 128, 9 - 16 (1971)

3. E.S. Evtukh, PhD Thesis (Bryansk, 2014)

4. V. Meshcherjakov, Proceedings of the 2nd Miniconference on Contact Mechanics and Wear of Rail (Wheel Systems. Budapest, 29-31 July, 1996)

5. M.F. Verigo, Interaction of the track and rolling stock (Moscow, Transport, 1986)

6. A.Ya. Kogan, Calculations of the railway track for vertical loading. Scientific papers of VNIIZhT, 502. (1993)

7. S.V. Vershinsky, V.N. Danilov, V.D. Khusidov, Dynamics of the car: A textbook for universities of rail.transp (Moscow, Transport, 1991)

8. M. P. Pakhomov, PhD Thesis (Moscow, MIIT, 1958)

9. Ya. G. Panovko., I.I. Gubanova, Stability and oscillations of elastic systems: Modern concepts, paradoxes and errors. (Moscow, Nauka, 1987)

10. A.N. Krylov, On some differential equations of mathematical physics M.-L.: GITTL., 1950. 359 p.

11. V. Ya. Shevchenko, PhD Thesis (Omsk, OmGUPS, 1987)

12. Ya. Z. Tsypkin, Fundamentals of the Theory of Automatic Systems (Moscow, Nauka, 1977)

13. V.A. Nekhaev, N.V. Zakernichnaya, Collection of abstracts of deposited manuscripts. Inv. B6541, 83. CMSI of the Ministry of Defense of the RF (2008)

14. V. A. Nekchaev, V.A. Nikolaev, Synthesis of invariant vibration protection system (theory and practice) / Nonlinear vibration problems (DWH-Polish Sientific Publishers. Warszawa, 1993)

15. I.I., Galiev, V.A. Nekhaev, V.A. Nikolaev, Methods and means of vibration protection of railway vehicles. (Moscow, Educational and Methodological Center for Education at the Railway Transport, 2010) 